\title{
EVALUACION DE CALIDAD DEL DESEMPEÑO DOCENTE EN LA CATEDRA DE SALUD DEL ADULTO Y ANCIANO EN LA FACULTAD DE ENFERMERIA DE LA UNIVERSIDAD NACIONAL SAN LUIS GONZAGA DE ICA, 2015
}

Evaluation of the quality of teaching performance in the adult and elderly health professorship in the nursing facultu of the National University San Luis Gonzaga from Ica, 2015

Rosa Herlinda Hernández Onofra ${ }^{1,3, a, c}$, Amanda María García Aquije ${ }^{3, a, c}$, M.C. Juana Maribel Arroyo Hernández ${ }^{2, b}$

${ }^{1}$ Facultad de Enfermería de la Universidad Nacional San Luis Gonzaga de Ica

${ }^{2}$ Facultad de Medicina de la Universidad Nacional San Luis Gonzaga de Ica

${ }^{3}$ Hospital Augusto Hernández Mendoza - Essalud, Ica.

aLicenciada en Enfermería, ${ }^{b}$ Médico Cirujano, 'Magíster en Salud Pública,

\section{RESUMEN}

Objetivo: Evaluar la calidad del desempeño de los docentes de la cátedra de salud del adulto y anciano, en la Facultad de Enfermería de la Universidad Nacional San Luis Gonzaga de Ica. Material y métodos: Estudio cuantitativo, de corte transversal y descriptivo. Muestra de 78 estudiantes del quinto ciclo de estudio que cursaron la asignatura de enfermería en la salud del adulto y anciano periodo 2015-II. Para la recolección de datos se utilizó la técnica de encuesta y como instrumento se utilizó un cuestionario validado por juicios de expertos y con una confiabilidad de alfa de cronbach de 0,6. Resultados: Se observó que el 54\% (42/78) de estudiantes expresaron que la calidad de desempeño docente es regular, el $26 \%$ (20/78) lo calificaron como bueno y el $10 \%(8 / 78)$ como muy bueno; el 10\% (8/78) expresaron que el desempeño docente es malo. Conclusiones: La evaluación de la calidad del desempeño docente en la catedra de salud del adulto y anciano es en su mayoría calificado por los estudiantes como regular, lo que permite deducir que el docente no está utilizando una metodología que logre un aprendizaje significativo.

Palabras clave: Evaluación de calidad del desempeño docente.

\section{SUMMARY}

Objective: Evaluate the quality of the performance of the teachers of the adult and elderly health professors in the Faculty of Nursing of the National University San Luis Gonzaga de Ica. Material and methods: Quantitative study, cross-sectional, application. Sample of 78 students of the 5th cycle of study who attended the subject of nursing in the health of the adult and old period 2015-II. For the data collection, the survey technique was used and a questionnaire validated by expert judgment and with a cronbach alpha reliability of 0.6 was used as instrument. Results: It is observed that of the $100 \%$ (78) of students surveyed, 54\% (42) students expressed that the quality of teaching performance is regular, $26 \%(20)$ is good $10 \%$ (8) very good and 10 (8) expressed that teacher performance is very poor, so it could be deduced that it would be limiting the acquisition of knowledge and the timely development of skills and abilities which would generate a deficit in the achievement of the competences in the subject limiting in A future approach to comprehensive and optimal care of the adult and elderly patient, as well as the motivation to care. Conclusions: The evaluation of the quality of teaching performance in the adult and elderly health chair is regular, which makes it possible to deduce that the teacher is not using a methodology that achieves meaningful learning.

Key words: Quality evaluation of teaching performance. 


\section{INTRODUCCIÒN}

La educación en el Perú pasa por una crisis que se refleja en la calidad del proceso educativo, según el Ministerio de Educación (2004) (1), el status profesional, social y económico del docente se ha deteriorado progresivamente, perdiendo de esta manera el reconocimiento de su liderazgo en propuestas de cambios sociales y en la forma como adquiere, construye y reconstruye el conocimiento. Según la misma fuente, con respecto a la práctica pedagógica, la mayoría de docentes siguen utilizando ciertas estrategias desfasadas y obsoletas, como la enseñanza rígida y memorista, actitudes poco democráticas, escaso interés por el trabajo en equipo, transmitiendo información y no desarrollando competencias en los estudiantes. Asimismo, la formación de valores no corresponde a la necesidad de interiorizar criterios ético-morales y se hace evidente un uso inadecuado de las nuevas tecnologías de información y comunicación.

El problema fundamental del sistema educativo es la calidad, siendo el profesor uno de los elementos más importantes en la tarea educativa, por lo tanto, cualquier esfuerzo por mejorar la calidad educativa en nuestro país debe basarse en información fáctica acerca de sus características profesionales y personales, conocer lo que piensa, siente y hace el profesor en su actividad profesional y cuál es la calidad de su desempeño.

De acuerdo a los lineamientos de la nueva Ley Universitaria № 30220 (2), la acreditación de las carreras profesionales es una necesidad para que las mismas sigan existiendo; para esta finalidad, la calidad docente es uno de los indicadores, donde el alumno es considerado fuente de evaluación, del desempeño docente. En el contexto internacional la evaluación ha sido planteada como un medio para mejorar la calidad de la educación, en nuestro país, cada universidad ha tenido la libertad de definir sus propios criterios de evaluación, lo cual no ha sido suficiente para mejorar en forma sustantiva la calidad de la educación. Rueda y col (2010) (3), señalan que el concepto de evaluación sobresale en las políticas educativas y se asocia al concepto de la calidad de la educación, por ser un mecanismo de medición y establecimiento de indicadores del proceso educativo y que debido a esto las instituciones educativas otorgan mayor importancia a evaluar el desempeño de los estudiantes, docentes, y el currículo, entre otros aspectos importantes, donde la evaluación docente destaca por considerarse pieza clave en el proceso de enseñanza.

La información sobre el desempeño docente, es valiosa para muchos investigadores, quienes se interesan en conocer los factores clave para la mejora del aprendizaje y la calidad de los servicios educativos, los nuevos escenarios en el que se desarrollan los estudiantes de hoy obligan a crear ambientes de aprendizaje que los preparen para asumir responsabilidades en un mundo globalizado, en rápido y constante cambio tanto social, como económico y tecnológico, este constante cambio en el que se desarrolla la tarea educativa nos obliga a analizar el desempeño de los docentes, y su capacitación permanente, para valorar su impacto y establecer mejoras. Quichca (2012) (4), considera que la excelencia académica es una meta que constituye uno de los elementos de mayor importancia y controversia en la educación superior contemporánea; y que su determinación se encuentra estrechamente vinculada entre otros al desempeño docente y que la calidad académica no es una abstracción, sino un referente social e institucional.

El presente trabajo de investigación es motivado por la necesidad que plantea la Ley Universitaria $N^{\circ} 30220$ (2), respecto de que las universidades forman parte de un sistema de calidad, siendo la labor del docente, uno de los conceptos a los que se le concede mayor énfasis, considerado como factor muy importante para el progreso y mejora del 
sistema educativo. Por lo tanto es motivo de especial atención la evaluación del desempeño.

Muñoz, Ríos y Abalde (2002) (5), afirman que el problema con la evaluación de la docencia surge generalmente porque tiene consecuencias escasas o nulas para la mejora de la calidad, tanto en la enseñanzaaprendizaje, como para el propio profesor, y por qué en la mayoría de los casos se lleva a cabo bajo un enfoque sancionador $y$ fiscalizador, que provoca la negación, rechazo y aversión de los docentes. Otra problemática que surge en torno a la evaluación de la docencia, es que hasta ahora el medio más empleado para realizarla han sido los cuestionarios de opinión de los alumnos, tomándolos como la mejor fuente de información; no obstante en términos generales, se considera que pueden estar cargadas de subjetividad, e influidas por diversos factores, y sesgos. (Sánchez y Domínguez 2008) (6).

En la Universidad Nacional San Luis Gonzaga de Ica, y en la Facultad de Enfermería, de las evaluaciones realizadas a docentes, los resultados han servido para el mejoramiento de la calidad de la educación, acciones encaminadas a propiciar su desarrollo futuro implementando un sistema de evaluaciones para cumplir con la Ley Universitaria. En este marco la presente investigación tuvo como objetivo: Evaluar la calidad del desempeño docente de la cátedra de salud del adulto y anciano, en la Facultad de Enfermería de la Universidad Nacional San Luis Gonzaga de Ica, 2015.

\section{MATERIAL Y MÉTODOS}

El presente estudio de investigación por la naturaleza de su problema es de tipo cuantitativo, de corte transversal ya que los datos fueron obtenidos en un determinado periodo de tiempo y de nivel aplicativo ya que permitió determinar el nivel de satisfacción de los estudiantes de enfermería sobre la enseñanza en la asignatura Enfermería en salud del adulto y anciano, y cuyos resultados contribuirán en la mejora de la calidad de la enseñanza aprendizaje que se brinda a los estudiantes de Enfermería de la Universidad Nacional San Luis Gonzaga de Ica.

La Muestra lo constituyeron en su totalidad los 78 estudiantes de pregrado del 50 ciclo de estudio que cursaron la Asignatura Enfermería en la Salud del Adulto y Anciano.

La técnica utilizada fue la encuesta y el instrumento una escala de Likert modificada, el instrumento se diseñó en forma estructurada de acuerdo a las dimensiones e indicadores que constan de las siguientes partes: presentación, introducción (donde se da a conocer la finalidad del estudio las instrucciones y el contenido propiamente dicho donde se incluyeron 29 enunciados entre positivos y negativos divididos en cuatro áreas: A. Enseñanza, B. Características personales del docente, C. Responsabilidad, y D. Tutoría. Relacionada a la enseñanza en la asignatura Enfermería en salud del adulto y anciano, dándole un puntaje donde el nivel de satisfacción es como sigue:

- Excelente: $90-100 \%$

- Muy Bueno: 80-89\%

- Bueno 71-79\%

- Regular: 61 a70\%

- Malo: 51 a $60 \%$

- Muy Malo: $50 \%$ a menos

\section{RESULTADOS.}

Respecto a la evaluación del desempeño docente de la catedra de Salud del Adulto y Anciano, se observa que, del total de estudiantes encuestados, el 54\% (42/78) estudiantes expresaron que la calidad de desempeño docente es regular, el $26 \%(20 / 78)$ es bueno el $10 \%$ (8/78) muy bueno y $10 \%$ $(8 / 78)$ expresaron que el desempeño docente es muy malo.

Tabla 1. "Resultado global de la evaluación del desempeño docente de la catedra de Salud del Adulto y Anciano". Facultad de Enfermería de la Universidad Nacional San Luis Gonzaga de Ica 2015 


\begin{tabular}{lcc}
\hline $\begin{array}{l}\text { Expresiones } \\
\text { Cualitativas }\end{array}$ & Frecuencia & Porcentaje \\
\hline Excelente & 0 & 0 \\
\hline Muy bueno & 8 & $10 \%$ \\
\hline Bueno & 20 & $26 \%$ \\
\hline Regular & 42 & $54 \%$ \\
\hline Malo & 8 & $10 \%$ \\
\hline Muy malo & 0 & 0 \\
\hline TOTAL & 78 & $100 \%$ \\
\hline
\end{tabular}

Gráfico 1. "Resultado global de la evaluación del desempeño docente de la catedra de Salud del Adulto y Anciano". Facultad de Enfermería de la Universidad Nacional San Luis Gonzaga de Ica 2015

\section{Calidad del Desempeño}

\section{Docente}

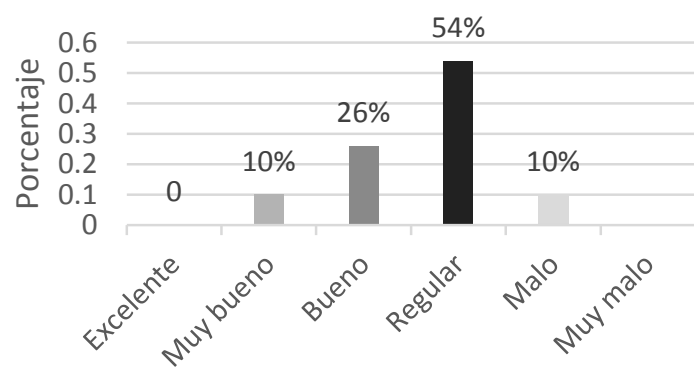

Expresiones cualitativas

\section{DISCUSIÓN}

El proceso de enseñanza aprendizaje tiene un carácter bilateral ya que existe relación entre la actividad del docente enseñar y la del alumno aprender en esta interacción es esencial que exista confianza, respeto, empatía y tolerancia para lograr una aceptación recíproca y constructiva.

Los factores pedagógicos incluyen la concepción de cómo se consigue que las personas aprendan cosas, y a partir de eso modifiquen su comportamiento. Es así que la pedagogía de transmisión enfatiza que las ideas y los aspectos de aprendizaje son los más importantes en la educación, y como consecuencia, la experiencia fundamental en el alumno para alcanzar sus objetivos trazados en las asignaturas. Estamos acostumbrados a la práctica de la enseñanza en un estilo donde el docente es autoritario y vertical en la transmisión de nuevos conocimientos y donde el estudiante, con poco esfuerzo, no pueda desarrollar habilidades intelectuales como: observación, análisis, evaluación, comprensión, trayendo como consecuencias entre otros elevada absorción de información, hábito de tomar notas y memorizar, algunas veces pasividad y falta de actitud crítica, profundo "respeto" por las fuentes de información (profesores, libro), distancia entre la teoría y la práctica, desvinculación con la realidad, prevalencia de exposiciones orales y donde la evaluación pretende la reproducción exacta y memorística por parte del estudiante.

La pedagogía de condicionamiento enfatiza los resultados de comportamiento es decir las manifestaciones empíricas y operacionales del intercambio de conocimientos, actitudes y destrezas. Así se concentra en el modelo de conducta mediante un juego eficiente de estímulos y recompensas capaz de "condicionar" al alumno a emitir respuestas deseadas por el profesor, pudiendo traer como consecuencias un estudiante pasivo, respuestas mecanizadas, no cuestionamiento de los objetivos ni el método, el docente es un técnico reproductor de la verdad científica, los procedimientos de enseñanza priorizan los sistemas instrumentales: en base a objetivos preestablecidos. La evaluación enfatiza la objetividad y la productividad; sin embargo, a veces, el educando se encuentra desarticulado de la realidad, no problematiza ni se le pide un análisis crítico de la misma, no tiene oportunidad de criticar los mensajes (contenidos temáticos) del programa de enseñanza establecidos, el tipo y oportunidad de los refuerzos, demostraciones y re demostraciones son determinados por el programador del sistema y con tendencia al individualismo y a la competitividad.

En esta investigación estamos analizando los resultados para darnos cuenta que el alumno no está conforme con las prácticas de la metodología de la enseñanza impartidas en la asignatura de Salud del Adulto y Anciano, lo que obliga al docente a asumir su rol y aceptar la problemática existente, para lograr que el alumno participante $y$ agente de la 
transformación social pueda detectar los problemas reales y buscar para ellos soluciones originales y creativas.

Según el syllabus, de la Asignatura Enfermería en Salud del Adulto y Anciano, para la estrategia metodológica se emplea el enfoque pedagógico innovador, con una metodología activa participativa, de tal manera que el alumno asume un rol protagónico en su aprendizaje, desarrollando su capacidad de análisis, síntesis e investigación, es decir ser un educando proactivo.

Al respecto, el Dr. Rubén Edel Navarro (2004) (7), señala que los nuevos modelos educativos demandan que los docentes transformen su rol de expositores del conocimiento al de monitores del aprendizaje y los estudiantes de espectadores del proceso de enseñanza, al de integrantes participativos, propositivos $y$ críticos en la construcción de su propio conocimiento.

Por otro lado el syllabus también señala que la práctica se desarrolla con la participación activa de los alumnos, forjadores de su aprendizaje, y que cuentan con la supervisión permanente, orientación y evaluación constante de los docentes de práctica, quienes son los que monitorean los logros del estudiante a fin de reforzar aquellos aspectos que considere deficientes. Por otro lado las prácticas se desarrollan a través de demostraciones, re demostraciones, utilizando el enfoque pedagógico de la problematización.

En esta perspectiva la enseñanza existe para el aprendizaje de forma productiva y racional, su función se encamina a estimular, dirigir y controlar el aprendizaje para que el estudiante participe en forma voluntaria y consciente en su formación profesional y adquiera de esta manera por su propio esfuerzo los conocimientos, actitudes y aptitudes que le permitan alcanzar los objetivos dados y ocupar un lugar en el grupo social al que pertenece.

Es así que la parte más importante para la ejecución de las actividades de aprendizaje están dadas por la formulación de los objetivos educativos, los cuales describen lo que el educando será capaz de hacer o demostrar una vez que haya completado con éxito una o más experiencias de aprendizaje. Estos objetivos deben ser enunciados en forma clara y precisa para que el alumno pueda identificar los logros que se espera de él; si no se tiene claro los objetivos al finalizar un proceso de enseñanza aprendizaje, no podrá evaluarse los logros alcanzados dificultando que se pueda realizar los reajustes o cambios necesarios para mejorar la enseñanza.

En la misma óptica, Nereci Imidio (1990) (8), refiere que estudiantes y profesores tengan conciencia de la dirección de sus esfuerzos; es decir tener claros y precisos sus objetivos y darse a conocer al principio del curso, pero el docente debe tenerlos en cuenta durante toda la sesión y recordarles a los alumnos; así se evitaran desviaciones del propósito de la clase.

De otro lado los temas tratados son parte del cuerpo de conocimientos empleados por docentes y educandos en el proceso enseñanza aprendizaje siendo valioso como un medio para adquirir habilidades y actitudes mentales necesarios para descubrir nuevos conocimientos. Los contenidos deben ser claros, actualizados y estructurados de tal manera que permitan la integración, continuidad y secuencialidad, además de guardar relación con la práctica y cumplir con lo establecido para que sean más fácilmente comprensibles asimiladas por los alumnos cubriendo así sus expectativas de aprendizaje. Así mismo el educador no sólo debe realizar una buena presentación que incluya de manera ordenada los aspectos del tema a tratar, bibliografía actualizada, guardar relación con la realidad y contenidos de la práctica, sino que también denoten preparación del docente.

Por otro lado, las estrategias comprenden las actividades que deben desarrollar el alumno y el docente dentro de una asignatura dependiendo de los objetivos que se desean 
alcanzar, cada una de estas actividades debe desarrollarse también de acuerdo a la disciplina y los recursos disponibles; para que se establezca una comunicación didáctica que transmita el mensaje educativo deseado.

En este sentido, la evaluación, debe ir en relación a los objetivos planteados y acostumbrar al estudiante a fijar su atención en los puntos importantes de los expuestos, evitando la repetición mecánica de todo lo que dijo el docente o se expuso del libro.

Según Candela Linares (2012) (9), el docente debe ocuparse constantemente de la evaluación del trabajo de sus alumnos como también monitorizar sus logros a fin de reforzar aspectos deficientes en un tiempo útil.

De los resultados obtenidos del total de 78 encuestados, todos son adultos jóvenes y en su mayoría del sexo femenino sólo 4 son masculino. Al realizar un análisis en base a los hallazgos se puede deducir que probablemente esto se deba a que el docente no seleccione adecuadamente las experiencias de aprendizaje ni destaca aspectos importantes de acuerdo al tema a tratar,, de manera que, la transmisión del mensaje no siempre es el esperado; una elevada información impartida por el docente, comunicación vertical del profesor hacia el educando, implicaría pues, realizar mejoras en la utilización de estrategias de aprendizaje así como reforzar las ya existentes, sobre todo porque esta enseñanza impartida debe darse en forma óptima al estudiante por la importancia que ella tiene en la formación de los futuros profesionales.

En cuanto a la evaluación del desempeño docente en la catedra de salud del adulto y anciano de la facultad de enfermería el 54\% (42) tienen una opinión que la calidad de enseñanza es regular, el $26 \%(20)$ es muy buena y $10 \%$ (8) es muy malo y $10 \%$ es muy buena, por lo que se puede deducir que probablemente el docente comúnmente actúa como transmisor de conocimientos y el alumno como un receptor pasivo en la mayoría de las clases, que se limita a tomar apuntes de forma mecánica favoreciendo un aprendizaje memorístico, existiendo también una comunicación vertical y unidireccional, el profesor no detecta aspectos importantes del tema a tratar y no ayuda a participar activamente en clase; presentando, el estudiante, deficientes bases conceptuales que son falencias, frente a su paciente; por otro lado, no se suele informar los objetivos de la clase, por lo que sería imprescindible que el docente analizara las probables causas que estarían determinando este resultado, ya que el presente estudio no incluye un abordaje de las razones que tienen los educandos al emitir un juicio desfavorable con respecto al desempeño del docente en la asignatura.

\section{CONCLUSIONES}

Respecto a la evaluación de la calidad del desempeño docente en la catedra de Salud del adulto y anciano, se determinó que la evaluación de la calidad es en su mayoría regular $54 \%$, según el instrumento aplicado a los estudiantes del quinto ciclo, por lo que se podría deducir que se estaría limitando la adquisición de conocimientos y el desarrollo oportuno de habilidades y destrezas lo que generaría déficit en el logro de las competencias en la asignatura limitando en un futuro el abordaje del cuidado integral y optimo del paciente adulto y anciano, así como la desmotivación para su atención.

Respecto a la evaluación del desempeño docente en la catedra de salud del adulto y anciano es regular considerado como lo que permite deducir que el docente no está utilizando una metodología que logre un aprendizaje significativo, por lo que existen deficiencias en el logro de competencias procedimentales, lo que generaría inseguridad en su desempeño con riesgo a improvisaciones con deficientes bases conceptuales que son falencias para el futuro ejercicio de la profesión , frente a su paciente; por otro lado A todo ello podemos decir que se 
rechaza la hipótesis del estudio y se acepta la hipótesis nula.

\section{RECOMENDACIONES}

- Que las autoridades de Enfermería formulen o diseñen estrategias para mejorar los métodos de enseñanza aprendizaje en los cursos de carrera especialmente en la asignatura Enfermería en salud del adulto y anciano.

- Realizar estudios sobre el tema, ampliado hacia los factores que intervienen en la enseñanza teórico-práctico a fin de encontrar los puntos críticos del proceso enseñanza aprendizaje en los estudiantes de enfermería.

- Implementar un comité de monitoreo y supervisión del cumplimiento de las actividades de los docentes pero con oportunidad.

\section{Correspondencia:}

Mg. Rosa Hernández Onofra

Correo electrónico: rosaherono@hotmail.com

\section{REFERENCIAS BIBLIOGRÁFICAS}

1. Ministerio de Educación. Propuesta Preliminar del Sistema Nacional de Formación Continúa de docentes. Documento de trabajo de la Dirección Nacional de Formación y Capacitación Docente. Lima, Perú, 2004

2. Diario el Peruano. Normas Legales: Ley Universitaria $N^{\circ} 30220$ del Perú. Lima, Perú, 2014.

3. Rueda M, Luna E. La evaluación del desempeño docente en las universidades públicas de México. México. Primer coloquio nacional de evaluación del desempeño docente en educación superior. 2010. p7-25.

4. Quichca Torres G. Relación entre la calidad de gestión administrativa y el desempeño docente según los estudiantes del I al VI ciclo 2010 - I del Instituto superior particular "La Pontificia" del distrito Carmen
Alto Provincia de Huamanga. Ayacucho, Perú, 2011.

5. Muñoz J, Ríos M, Abalde E. Evaluación Docente vs Evaluación de la calidad. México. 2002. Ed RELIEVE. 8, 103-134.

6. Sánchez S, Domínguez A. Elaboración de un instrumento de viñetas para evaluar el desempeño docente. México. Rev Mexicana de Investigación Educativa, 2008. 13(37): 625-648

7. Navarro R. El concepto de enseñanza aprendizaje. Sociedad Mexicana de Psicología y de la Red lberoamericana de Investigación sobre cambio y eficacia escolar. Veracruz, México. 2004.

8. Nerici, l. metodología de la enseñanza. $4 a$ ed. México. Ed Kapeluz mexicana: 1990.

9. Candela H. Investigación referente a la relación entre la transposición didáctica y el rendimiento académico. Lima, Perú. 2012. Rev. Investigación Educativa 16 (29): 125-132.

Recibido: 15/11/2016

Aprobado para Publicación: 16/05/2017 